\title{
Parâmetros Genéticos para as Produções de Leite no Dia do Controle e da Primeira Lactação de Vacas da Raça Holandesa ${ }^{1}$
}

\author{
Cláudio Manoel Rodrigues de Melo², Irineu Umberto Packer ${ }^{3}$, Cláudio Nápolis Costa ${ }^{4}$, Paulo \\ Fernando Machado ${ }^{3}$
}

\begin{abstract}
RESUMO - Foram utilizados 263.390 registros de produção de leite no dia do controle (PDC) de 32.448 primeiras lactações de vacas da raça Holandesa com parto no período de 1991 a 2001, para estimar componentes de variância e parâmetros genéticos utilizando um modelo animal e a metodologia REML. Os dados de produção foram coletados pelo Serviço de Controle Leiteiro da Associação Brasileira de Criadores de Bovinos da Raça Holandesa e suas afiliadas estaduais e disponibilizados pela Embrapa Gado de Leite. O modelo para ajuste da produção de leite até 305 dias (P305) incluiu os efeitos fixos de rebanho-ano de parto, época do parto e idade da vaca ao parto, com termos linear e quadrático, e os efeitos aleatórios de animal e erro. Os mesmos efeitos foram incluídos no modelo para as produções de leite no dia do controle (PDC) tanto sob modelo uni- e bi-caráter como sob modelo de repetibilidade (MRS), exceto para o efeito fixo de grupo contemporâneo, definido por rebanho-ano-mês do controle. Alternativamente, ajustou-se um segundo modelo de repetibilidade (MRF), que além dos efeitos presentes no MRS, incluiu as covariáveis que descrevem a curva da lactação: dias em lactação (DEL)/305 e $\ln (305 / \mathrm{DEL})$ com termos linear e quadrático. As estimativas de $\hat{h}^{2}$ para as PDC, com MRS e MRF foram 0,43 e 0,30, respectivamente. As estimativas de herdabilidade $\left(\hat{h}^{2}\right)$ para as PDC variaram de 0,22 (PDC1) a 0,36 (PDC4) com o modelo uni-caráter. Para o modelo bicaráter, as estimativas variaram de 0,23 (PDC1) a 0,33 (PDC3 e PDC4). Em ambos os modelos, observaram-se valores inferiores de $\hat{h}^{2}$ no início e no fim do período de lactação. A estimativa $\hat{h}^{2}$ para a P305 com o modelo uni-caráter foi 0,27, enquanto, pelo modelo bi-caráter, estas estimativas variaram de 0,27 a 0,30 . As correlações genéticas $\left(\mathrm{r}_{\mathrm{g}}\right)$ entre as PDC e a P305 foram altas, variando de 0,86 (PDC1 e P305) a 0,99 (PDC3 e P305). Estimativas de herdabilidade maiores para as PDC que para a P305 e a alta correlação com a mesma indicam potencial de uso das PDC nas avaliações genéticas de animais da raça Holandesa no Brasil. Embora predominantemente altas, as estimativas de $r_{g}$ entre as PDC não foram homogêneas $(0,64-1,0)$; entretanto as maiores freqüências foram para valores próximos ou iguais a 1 . Assim, modelos de regressão aleatória devem ser também avaliados para se concluir sobre a melhor utilização das PDC da raça Holandesa no Brasil.
\end{abstract}

Palavras-chave: análise bi-caráter, componentes de variância, modelo de repetibilidade, parâmetros genéticos, produções de leite do dia do controle

\section{Genetic Parameters for Test Day and Lactation Milk Yields of First Lactation Holstein Cows}

\begin{abstract}
Covariance components for test day records and lactation milk yield using 263.390 records of 32.448 first lactation Holstein cows, were estimated using animal models by REML. Besides the lactation model, two alternative repeatability models (RM) were analyzed. Lactation model included fixed effects of herd-year-season and age of cow with linear and quadratic terms, and random effects of animal and error. The first model for test-day yield (RMF) included the same effects, but fixed effect of contemporary group, defined as herd-year-month of test. Alternatively another model for test-day yield (RMF) used a logarithmic polynomial sub-model for the shape of the lactation curve. Heritability for lactation yield (0.27) was smaller than those estimated by RMF and RMS, 0.30 and 0.43 , respectively. Heritability estimates for univariate (0.22-0.36) and bivariate models (0.23-0.33) for test day milk yields were found to be smallest during early and late lactation. Heritability estimate for lactation milk yield when estimated by univariate model ( 0.27$)$ was smaller than estimates obtained by bivariate models (0.27-0.30). Genetic correlations were higher between consecutive test days than between test days in the beginning and end of lactation. Larger heritability estimates for test day models and large genetic correlations between test day and lactation yield (0.86-0.99) indicate a potential use of test day records in genetic evaluations.
\end{abstract}

Key Words: genetic evaluation, genetic parameters, milk yield, multivariate analysis, selection, test day model, variance components

\section{Introdução}

A produção de leite até 305 dias (P305) é o indicador zootécnico geralmente utilizado para as comparações entre vacas nas decisões de manejo e descarte nos rebanhos leiteiros. A P305 é calculada usando os registros de produção provenientes dos controles leiteiros, que são realizados em intervalos aproximadamente mensais.

A utilização da P305 nas comparações entre os animais requer o seu ajuste para os efeitos de reba-

\footnotetext{
${ }^{1}$ Pesquisa financiada pela Fundação de Amparo à Pesquisa do Estado de São Paulo (FAPESP), processo 99/03838-6 e pelo Prodetab/ Embrapa, integrante do Projeto 054-02/99.

2 Professor da UFSC. Departamento de Aqüicultura - AQI, CEP: 88040-900, Florianópolis, SC, Brasil (cmrmelo@cca.ufsc.br).

3 Professores do Departamento de Zootecnia - USP/ESALQ, CEP: 13418-900, Piracicaba, SP, Brasil (iupacker@esalq.usp.br).

${ }^{4}$ Pesquisador da Embrapa Gado de Leite, CEP: 36038-330, Juiz de Fora, MG, Brasil (cnc8@cnpgl.embrapa.br).
} 
nho, ano de parto, época de parto, duração da lactação, entre outros. Entretanto, estes efeitos são considerados em termos médios, pois são definidos com base na data do parto.

Uma alternativa à utilização da $\mathrm{P} 305$ seria o uso da produção de leite no dia do controle (PDC). Este procedimento possibilita um ajuste mais preciso para os efeitos de ambiente temporário, visto que ele permite ajustar para os efeitos presentes em um dado dia ou mês de controle (Ptak \& Schaeffer, 1993; Swalve, 1995), viabiliza o uso de mais dados de um mesmo animal e o uso de dados de animais com lactações em andamento ou parciais, sem necessidade de cálculo da P305, possibilitando-se avaliações genéticas mais freqüentes e redução do intervalo de gerações (Olori, 1997).

Em virtude do uso de mais dados de uma mesma vaca, as avaliações com base nas PDC, quando comparados àquelas utilizando a $\mathrm{P} 305$, podem ser mais confiáveis, o que é evidenciado pelas maiores estimativas de herdabilidade obtidas para as PDC (Visscher \& Goddard, 1995; Strabel \& Szwaczkowski, 1997). Neste contexto, Ptak \& Schaeffer (1993) indicam a necessidade de considerar, no modelo de ajuste, a forma da curva de lactação, que pode ser incorporada usando coeficientes de regressão do caráter, em função do número de dias em lactação (DEL).

Outra consideração sobre os modelos para o ajuste das PDC refere-se à formação dos grupos contemporâneos. Danell (1982) recomenda o agrupamento de todas as vacas de determinado rebanho-data do controle. Em vários estudos (Reents et al., 1995; Swalve, 1995; Pösö et al., 1998; Rekaya et al., 1999), as estimativas de herdabilidade foram maiores quando os grupos contemporâneos foram definidos como rebanho-data do controle que quando definidos como rebanho-estação de parto. Isto também é observado quando se compara as estimativas de herdabilidade obtidas para as PDC àquelas obtidas para a P305 (Swalve, 1995; Strabel \& Szwaczkowski, 1997; Ferreira et al., 2003), sugerindo maior confiabilidade nas estimativas dos valores genéticos dos animais. Neste contexto, o uso de modelos para o ajuste das PDC em substituição aos modelos para o ajuste da P305 tem sido investigado por vários autores (Van Tassell et al., 1992; Reents, et al., 1995; Rekaya et al., 1995; Wiggans \& Goddard, 1997; Vargas et al., 1998).

Entre as alternativas para estimar componentes de variância e parâmetros genéticos adotando-se as PDC, o ajuste do modelo de repetibilidade (MR) caracteriza-se como a mais simples. O MR assume variâncias genéticas aditivas e de ambiente permanente constantes ao longo da lactação e correlações genéticas e de ambiente permanente entre as produções de uma mesma vaca iguais a um. Alguns estudos têm mostrado que correlações genéticas entre as PDC, dentro da primeira lactação, são próximas a um (Gadini, 1997; Gadini et al., 1998; Machado et al., 1998, Rekaya et al., 1999).

Pode-se, também, considerá-las como caracteres correlacionados e, então, analisá-las como medidas repetidas. Embora não assuma nenhuma estrutura de covariância entre os registros sucessivos, este procedimento é computacionalmente mais exigente, e, mesmo assim, é necessário conhecer a magnitude das covariâncias entre as PDC na lactação e entre as covariâncias e a P305, para avaliar a potencialidade e a estratégia de sua utilização nos procedimentos de seleção.

Alguns estudos desta natureza (Swalve, 1995; Machado, 1997; Rekaya et al., 1999) têm permitido concluir que estimativas de herdabilidades são menores para as produções do início e fim do período de lactação, em razão de menores estimativas de variância genética aditiva e de maiores estimativas de variâncias residuais para as PDC nestes períodos da lactação. De modo geral, estes resultados sugerem que as PDC podem ser usadas na seleção dos animais em substituição à P305. Entretanto, a escolha dos controles a serem utilizados deve considerar as estimativas de herdabilidade para as PDC e as estimativas de correlação genética entre as PDC e a P305. Assim, as PDC que apresentam maiores herdabilidades e maiores correlações genéticas com a P305 seriam as preferidas nos processos de seleção (El Faro, 2002).

Os mesmos estudos têm mostrado que as correlações genéticas entre as PDC e P305 são superiores no período intermediário da lactação quando comparadas àquelas do período inicial e do período final da lactação (Swalve, 1995; Rekaya et al., 1999), embora Rekaya et al. (1995) e Machado (1997) tenham relatado estimativas de correlações genéticas superiores entre PDC e P305 no início e fim da lactação.

Objetivou-se, neste estudo, estimar componentes de covariância e parâmetros genéticos, empregando modelos de repetibilidade, uni e bi-caráter, para o ajuste das produções de leite no dia do controle e para a produção de leite até 305 dias de primeiras lactações de vacas da raça Holandesa. 


\section{Material e Métodos}

\section{Dados}

Os registros de produção de leite foram coletados sob a supervisão do Serviço de Controle leiteiro (SCL) da Associação Brasileira de Criadores de Bovinos da Raça Holandesa (ABCBRH) e de suas filiadas estaduais e disponibilizados pelo Arquivo Zootécnico Nacional (AZN), gerenciado pela Embrapa Gado de Leite. Foram utilizados os dados de 39.048 lactações de 531 rebanhos dos estados de São Paulo (SP), Minas Gerais (MG), Rio Grande do Sul (RS), Santa Catarina (SC) e Espírito Santo (ES) controladas no período de 1991 a 2001.

Foram definidas quatro épocas de parto: 1 - janeiro a março; 2 - abril a junho; 3 - junho a setembro e 4 - outubro a dezembro. O grupo de contemporâneos (GC) foi definido como rebanho-ano-mês do controle nos modelos para ajuste das PDC e rebanho-ano de parto no modelo para ajuste da $\mathrm{P} 305$ e, em ambos, aplicou-se a restrição de que cada GC deveria conter pelo menos quatro vacas de 18 a 48 meses de idade.

Assim, no ajuste do modelo para P305, foram usados dados de produção de leite de 28.437 vacas pertencentes a 467 rebanhos. Para ajuste dos modelos para PDC, foram utilizadas somente as produções, coletadas entre cinco e 305 dias após o parto, de vacas com pelo menos três controles de produção, resultando em 32.448 primeiras lactações, com 263.390 registros de produção de leite provenientes de controles mensais, para análise.

$\mathrm{O}$ arquivo contendo dados de genealogia dos animais, para a estruturação da matriz de coeficientes de parentesco (A), foi caracterizado pela inclusão de 3.737 registros de touros aos 41.069 registros de vacas. Entretanto, animais não informativos (i.e., ambos pais desconhecidos e somente um descendente) foram eliminados. Assim, a matiz A incluiu 36.732 animais para o ajuste do modelo da P305 e 40.770 animais para o ajuste dos demais modelos.

Modelos

O modelo utilizado para o ajuste das PDC sob modelo uni- e bi-caráter ou para o ajuste da P305 foi:

$$
y_{i j k}=G C_{i}+E P O C A_{j}+\sum_{n=1}^{2} b_{n} x_{i j k}^{n}+a_{i j k}+e_{i j k}
$$

em que: $y_{i j k}$ é a PDC ou a P305; $G C_{i}$, o efeito do grupo contemporâneo $i$, formado pela combinação de rebanho-ano-mês do controle (PDC) ou de rebanhoano de parto (P305); $E P O C A_{j}$, a época do parto $j$; $b_{1}$ e $b_{2}$, os coeficientes de regressão linear e quadrático do caráter em função da idade da vaca ao parto; $x_{i j k}$, a idade da vaca ao parto, em dias; $a_{i j k}, \mathrm{o}$ efeito genético aditivo aleatório do animal $k$ pertencente ao rebanho-ano de parto $i$ e a época de parto $j$; e $e_{i j k}$, o erro aleatório associado a cada observação.

Para o ajuste das PDC sob modelo de repetibilidade (MRS), foi utilizado o seguinte modelo:

$$
y_{i j k}=R A M_{i}+E P O C A_{j}+\sum_{n=1}^{2} b_{n} x_{k}^{n}+a_{k}+p e_{k}+e_{i j k}
$$

em que: $y_{i j k}$ é a PDC da vaca $k$, que pariu na época $j$ e foi controlada no grupo $i ; R A M_{i}$, o efeito do $i$-ésimo grupo de controle formado pela combinação de rebanho, ano, mês do controle; $E P O C A_{j}$, a época do parto $j ; b_{1}$ e $b_{2}$, os coeficientes de regressão linear e quadrático da PDC, em função da idade da vaca ao parto; $x_{k}$, a idade da vaca $k$ ao parto, em dias; $a_{k}$, o efeito genético aditivo aleatório do animal $k$; $p e_{k}$, o efeito permanente da vaca $k$; e $e_{i j k}, o$ erro aleatório associado a cada observação.

Alternativamente, ajustou-se outro MR incluindo os coeficientes de regressão fixa da produção de leite, em função de covariáveis que descrevem a curva de lactação média da população (MRF):

$$
y_{i j k}=R A M_{i}+E P O C A_{j}+\sum_{n=1}^{2} b_{n} x_{k}^{n}+\sum_{n=1}^{4} q_{n} z_{n k}+a_{k}+p e_{k}+e_{i j k}
$$

em que: $q_{1}$ a $q_{4}$ são os coeficientes de regressão da PDC em função das covariáveis $z_{n k}$ que descrevem a curva da lactação, em que: em que: $z_{1 k}=c ; z_{2 k}=c^{2}$; $z_{3 k}=\ln (1 / c) ; z_{4 k}=(\ln (1 / c))^{2}$ e $\mathrm{c}=\mathrm{DEL} / 305$. Os demais efeitos são como descritos na eq. (2).

$\mathrm{Na}$ forma matricial, o modelo (1) pode ser escrito como:

$$
\mathbf{y}=\mathbf{X b}+\mathbf{Z a}+\mathbf{e}
$$

em que y é o vetor das observações; b é o vetor dos efeitos fixos; a é o vetor de efeito aleatório de animal; e é o vetor de efeito aleatório residual; e X e Z são as matrizes de incidência relativa às observações, para efeitos fixos e efeito aleatório de animal, respectivamente. Assumiu-se

$$
\left[\begin{array}{l}
\mathbf{a} \\
\mathbf{e}
\end{array}\right] \sim \mathrm{N}(\mathbf{0}, \mathbf{V}) ; \quad \mathbf{V}=\left[\begin{array}{l}
\mathbf{a} \\
\mathbf{e}
\end{array}\right]=\left[\begin{array}{cc}
\mathbf{G} \otimes \mathbf{A} & 0 \\
0 & \mathbf{R} \otimes \mathbf{I}_{\mathbf{N}}
\end{array}\right], \mathrm{em}
$$


que: $\mathbf{G}$ é a matriz de covariância genética aditiva; $\mathrm{R}$, a matriz de covariâncias residuais; A, a matriz de coeficientes de parentesco entre os animais; $\mathbf{I}_{\mathbf{N}}$, uma matriz identidade de ordem igual ao número de animais e $\otimes$, o operador de produto direto entre matrizes.

Da mesma forma, os MRF e MRS, eq. (2), podem ser descritos como:

$$
\mathbf{y}=\mathbf{X b}+\mathbf{Z a}+\mathbf{Z}_{\mathbf{D}} \mathbf{p}+\mathbf{e}
$$

em que $y, b, a, e, X, e \mathrm{Z}$ são como definido anteriormente; $\mathrm{p}$ é o vetor de efeitos permanentes de ambiente; e $\mathbf{Z}_{\mathbf{D}}$ é a matriz de incidência relativa ao efeito aleatório permanente de ambiente. Assumiu-se $\operatorname{var}(\mathbf{e})=\mathbf{I} s_{e}^{2}=\mathbf{R}, \operatorname{var}(\mathbf{p})=\mathbf{I} \boldsymbol{\sigma}_{p}^{2}, \operatorname{var}(\mathbf{a})=\mathbf{A} \boldsymbol{\sigma}_{a}^{2} \mathrm{e}$, portanto, $\operatorname{var}(\mathbf{y})=\mathbf{Z} \mathbf{A} \mathbf{Z}^{\prime} \boldsymbol{\sigma}_{a}^{2}+\mathbf{Z}_{\mathbf{D}} \mathbf{Z}_{\mathbf{D}}^{\prime} \boldsymbol{\sigma}_{p}^{2}+\mathbf{R}$. Os componentes de covariância foram estimados utilizando-se o programa REMLF90 (Misztal, 1999), baseado na metodologia de máxima verossimilhança restrita (REML), com o algoritmo de Maximização da Esperança (EM), e um processo de aceleração da convergência. Definiu-se como critério de convergência o valor do quadrado das diferenças entre estimativas consecutivas menor que $10^{-9}$.

Procedeu-se à decomposição canônica das matrizes de covariância genética $(\mathrm{G})$ e residual $(\mathrm{R})$ estimadas para as PDC com intuito de definir a provável ordem necessária de ajuste das PDC sob modelos de regressão aleatória.
A eficiência relativa de seleção (ER) baseada na produção dos 10 controles, na produção dos cinco primeiros controles, na produção de controles alternados de dois em dois meses (PDC1, PDC4, PDC7 e PDC10) ou, ainda, na produção de cada controle individualmente, em relação à seleção pela P305, foi calculada para determinar o ganho/perda resultante da seleção pelas produções do dia do controle em relação a P305. Definiu-se o agregado como $\mathbf{a}=30 \times \mathrm{PDC} 1+30 \times \mathrm{PDC} 2+\cdots+30 \times \mathrm{PDC} 10$, de forma a ter uma equivalência com a P305 (correlação esperada entre a e P305 igual a 1). A ER foi calculada como:

$$
\mathbf{E R}=\frac{\omega}{\sqrt{0,27}} \quad \text { em que } \quad \omega=\sqrt{\frac{\mathbf{a} \times \mathbf{G} \times \mathbf{P}^{-1} \mathbf{G} \times \mathbf{a}^{\prime}}{\mathbf{a} \times \mathbf{G} \times \mathbf{a}^{\prime}}}
$$

a correlação do agregado com a índice, $\mathrm{G}$ é a matriz de covariância genética aditiva ou parte dela (dependendo de quais controles estão sendo usados no cálculo da ER), P é a matriz de covariância fenotípica ou parte dela e 0,27 , a herdabilidade estimada para P305.

\section{Resultados e Discussão}

As informações sobre número de lactações e de controles, médias de produção em até 305 dias e nos controles, e número de animais considerados no estudo são apresentadas na Tabela 1.

Tabela 1 - Número de observações (N), médias e desvios-padrão (DP) para a produção de leite no dia do controle (PDC) e respectivos dias em lactação e para a produção de leite até 305 dias de lactação (P305)

Table 1 - Number of records (N), average and standard deviations (SD) for test day milk yield (YTD) and respective days in milk

\begin{tabular}{|c|c|c|c|c|c|}
\hline \multirow{2}{*}{$\begin{array}{l}\text { Controle } \\
\text { Test day }\end{array}$} & \multirow{2}{*}{$\mathrm{N}$} & \multicolumn{2}{|c|}{$\begin{array}{c}\text { Produção de leite }(\mathrm{kg}) \\
\text { Milk yield }(\mathrm{kg})\end{array}$} & \multicolumn{2}{|c|}{$\begin{array}{c}\text { Dias em lactação } \\
\text { Days in milk }\end{array}$} \\
\hline & & $\begin{array}{l}\text { Média } \\
\text { Average }\end{array}$ & $\begin{array}{l}\mathrm{DP} \\
S D\end{array}$ & $\begin{array}{l}\text { Média } \\
\text { Average }\end{array}$ & $\begin{array}{l}\mathrm{DP} \\
S D\end{array}$ \\
\hline $\begin{array}{l}\text { PDC1 } \\
Y T D 1\end{array}$ & 30.904 & 22,66 & 6,23 & 23 & 10 \\
\hline PDC2 & 31.308 & 24,58 & 6,46 & 55 & 12 \\
\hline PDC3 & 31.592 & 24,38 & 6,66 & 87 & 14 \\
\hline PDC4 & 30.460 & 23,82 & 6,76 & 119 & 16 \\
\hline PDC5 & 29.275 & 23,14 & 6,88 & 151 & 17 \\
\hline PDC6 & 28.201 & 22,30 & 6,87 & 183 & 19 \\
\hline PDC7 & 26.844 & 21,39 & 6,81 & 213 & 20 \\
\hline PDC8 & 24.649 & 20,48 & 6,75 & 244 & 19 \\
\hline PDC9 & 19.811 & 19,59 & 6,58 & 269 & 15 \\
\hline PDC10 & 10.346 & 18,92 & 6,48 & 291 & 9 \\
\hline $\begin{array}{l}\text { P305 } \\
Y 305\end{array}$ & 28.437 & $6.246,14$ & $1.932,13$ & - & - \\
\hline
\end{tabular}
and for 305-days milk yield (Y305) of first lactation Holstein cows 
As PDC determinam a forma típica da curva de lactação, com ligeiro aumento da produção inicial de cerca de $22 \mathrm{~kg}$ até o pico da lactação (período de 55 a 87 dias de lactação) com aproximadamente $24 \mathrm{~kg}$, decrescendo posteriormente até $19 \mathrm{~kg}$ de leite ao final da lactação. A variação entre as produções de leite foi homogênea (desvio-padrão médio de $6,65 \mathrm{~kg}$ de leite) nos controles da lactação.

$\mathrm{Na}$ Tabela 2, são apresentadas as estimativas dos componentes de variância e parâmetros genéticos para a P305 e para as PDC sob modelo de repetibilidade.

Tabela 2 - Estimativas de componentes de variância genética aditiva, permanente de ambiente, residual e fenotípica, herdabilidades e repetibilidade para a produção de leite até 305 dias (P305) e para as produções de leite no dia do controle (PDC) de primeiras lactações de vacas da raça Holandesa

Table 2 - Estimates of variance components for additive genetic, permanent environment, residual, and phenotypic effects, heritability and repeatability for test day milk yield (YTD) and 305-days milk yield (Y305) of first lactation Holstein cows

\begin{tabular}{lccc}
\hline $\begin{array}{l}\text { Efeitos/Componentes de variância } \\
\text { Effects/Variance components }\end{array}$ & & $\begin{array}{c}\text { Modelo } \\
\text { Model }\end{array}$ \\
\cline { 2 - 4 } & $\mathrm{P} 305^{1}$ & $\mathrm{MRS}^{2}$ & $\mathrm{MRF}^{3}$ \\
\hline $\begin{array}{l}\text { Genético aditivo } \\
\text { Additive genetic }\end{array}$ & 647.700 & 13,980 & 7,217 \\
$\begin{array}{l}\text { Permanente de ambiente } \\
\text { Permanent environmental }\end{array}$ & - & 7,934 & 8,139 \\
$\begin{array}{l}\text { Residual } \\
\text { Residual }\end{array}$ & 1.719 .000 & 10,430 & 8,558 \\
$\begin{array}{l}\text { Fenotípico } \\
\text { Phenotypic }\end{array}$ & 2.366 .700 & 32,344 & 23,914 \\
$\begin{array}{l}\text { Herdabilidade } \\
\text { Heritability } \\
\text { Repetibilidade }\end{array}$ & 0,27 & 0,43 & 0,30 \\
Repeatibility & - & 0,68 & 0,64 \\
\hline
\end{tabular}

${ }^{1}$ Modelo para P305, ${ }^{2}$ Modelo de repetibilidade, ${ }^{3}$ Modelo de repetibilidade com regressão fixa.

${ }^{1}$ Y305-day model, ${ }^{2}$ Repeatability model, ${ }^{3}$ Fixed regression repeatability model.

As estimativas de herdabilidade para as PDC $(0,43$ e 0,30$)$ foram maiores que aquela obtida para a P305 $(0,27)$, semelhantes às reportadas por Costa et al. (2000), Freitas et al. (2001) e Ferreira et al. (2003) e dentro dos limites $(0,22-0,49)$ observados na literatura (Costa et al., 1999; Machado et al., 1999; Torres et al., 1999).

Valores de herdabilidades superiores para as PDC em relação aos obtidos para a P305 foram também relatados por Strabel \& Szwaczkowski (1997) e Ferreira et al. (2003). Portanto, os resultados do presente estudo confirmam que os MR para o ajuste das PDC resultam em maiores estimativas de herdabilidade que os modelos para o ajuste da P305.

As estimativas dos componentes de variância diferiram entre os MR, com a maior diferença entre as variâncias genéticas $(6,763)$, seguidas daquela entre as variâncias residuais $(1,872)$, e uma diferença muito pequena entre os componentes do efeito permanente de ambiente $(0,205)$. A estimativa de herdabilidade $(0,43)$ obtida com o MRS foi maior que a $(0,30)$ encontrada por meio do MRF. A redução na estimativa da variância residual, pela inclusão das covariáveis que descrevem a forma da curva da lactação era esperada, no entanto, não se esperava a redução na estimativa da variância genética aditiva, pois os coeficientes que descrevem a forma da curva de lactação foram tomados como fixos.

As estimativas das variâncias obtidas com o ajuste dos MR foram semelhantes às reportadas por Ferreira et al. (2003) e encontram-se dentro dos limites apresentados na literatura (Reents et al. 1995; Swalve 1995; Olori, 1997; Pösö et al. 1998; Rekaya et al. 1999) para a variância genética $(12,70-2,77)$, para o efeito permanente de ambiente $(8,60-3,26)$ e residual $(7,40-3,80)$.

Os valores de herdabilidade $(0,30)$ e de repetibilidade $(0,64)$ obtidos com o ajuste do MRF, foram semelhan- 
tes aos reportados por Rekaya et al. (1999). Entretanto, o valor de herdabilidade de 0,43 , verificado no ajuste do MRS foi superior a 0,31, obtido por Pool et al. (2000). Estes valores $(0,43$ e 0,30$)$ também se assemelham às estimativas reportadas por Olori (1997) para modelos de repetibilidade que consideravam a forma da curva da lactação por meio de regressões polinomial quadrática, cúbica e de quarta ordem (MRF), ou na ausência destas regressões (MRS).

As estimativas dos componentes de variância, herdabilidades para as PDC, sob modelo de análise uni-caráter e para a P305 e ER, são apresentadas na Tabela 3.

As estimativas de variância genética aditiva para as PDC aumentaram do primeiro controle $\left(5,01 \mathrm{~kg}^{2}\right)$ até o quarto controle $\left(7,88 \mathrm{~kg}^{2}\right)$, reduzindo-se daí por diante até o último controle $\left(4,80 \mathrm{~kg}^{2}\right)$. As maiores estimativas foram obtidas para as PDC3 e PDC4. Ao contrário do observado para as variâncias genéticas, as estimativas das variâncias residuais foram superiores para as produções do início (PDC1) e do fim (PDC10) do período de lactação.

Os valores de herdabilidade para PDC aumentaram do início da lactação até a fase intermediária (quarto controle); a partir deste ponto, até o final da lactação, ocorreu diminuição destes valores (Figura 1). $\mathrm{O}$ maior valor das estimativas de herdabilidade para as PDC na fase intermediária da lactação ocorreu mais em função do aumento nas estimativas das variâncias genéticas que em função da redução das variâncias residuais. Estes resultados são similares aos obtidos por Olori (1997), Rekaya et al. (1999) e Ferreira et al. (2003), mas não aos apresentados por El Faro (2002), que obteve maior estimativa de herdabilidade para os controles do início e do fim do período da lactação. As estimativas de herdabilidade para as PDC foram geralmente superiores à obtida para a P305, exceto para as PDC1, PDC9 e PDC10 (Figura 1). Estes resultados também se assemelham aos reportados em vários outros estudos (Meyer et al., 1989; Swalve, 1995; Kettunen et al., 1998; Machado et al., 1998).

As estimativas de herdabilidade resultantes das análises bi-caráter (Tabela 4) foram semelhantes àquelas obtidas pelas análises uni-caráter e situaram-se dentro do intervalo de valores relatados por Rekaya et al. (1999) e Ferreira et al. (2003).

As estimativas das correlações genéticas e fenotípicas entre as PDC e entre estas e a P305 encontram-se na Tabela 5.

Tabela 3 - Estimativas dos componentes de variância genética aditiva $\left(\hat{\sigma}_{a}^{2}\right)$, residual $\left(\hat{\sigma}_{e}^{2}\right)$, fenotípica $\left(\hat{\sigma}_{p}^{2}\right)$ e de herdabilidades $\left(\hat{h}^{2}\right)$ para as produções de leite no dia do controle (PDC) e para a produção de leite até 305 dias (P305) de primeiras lactações de vacas da raça Holandesa, em análise uni-caráter, e as eficiências relativa de seleção (ER)

Table 3 - Estimates of genetic additive $\left(\hat{\sigma}_{a}^{2}\right)$, residual $\left(\hat{\sigma}_{e}^{2}\right)$, and phenotypic $\left(\hat{\sigma}_{p}^{2}\right)$, variance components and heritability $\left(\hat{h}^{2}\right)$ for test day milk yield (YTD) and for 305-days milk yield (Y305) of first lactation Holstein cows by univariate mode and relative selection efficiency (RE)

\begin{tabular}{|c|c|c|c|c|c|}
\hline $\begin{array}{l}\text { Controle } \\
\text { Test day }\end{array}$ & $\hat{\sigma}_{a}^{2}$ & $\hat{\sigma}_{e}^{2}$ & $\hat{\sigma}_{p}^{2}$ & $\hat{h}^{2}$ & $\begin{array}{l}\mathrm{ER} \\
R E\end{array}$ \\
\hline $\mathrm{PDC1}$ & 5,01 & 17,86 & 22,87 & 0,22 & 0,82 \\
\hline \multicolumn{6}{|l|}{ YTDI } \\
\hline PDC2 & 6,65 & 14,99 & 21,64 & 0,31 & 1,02 \\
\hline PDC3 & 7,55 & 14,19 & 21,74 & 0,35 & 1,11 \\
\hline PDC4 & 7,88 & 13,84 & 21,72 & 0,36 & 1,16 \\
\hline PDC5 & 6,77 & 14,73 & 21,50 & 0,32 & 1,08 \\
\hline PDC6 & 6,66 & 14,52 & 21,18 & 0,31 & 1,08 \\
\hline PDC7 & 6,65 & 14,25 & 20,90 & 0,32 & 1,08 \\
\hline PDC8 & 6,32 & 14,44 & 20,76 & 0,30 & 1,04 \\
\hline PDC9 & 5,34 & 15,47 & 20,81 & 0,26 & 0,94 \\
\hline PDC10 & 4,80 & 15,57 & 20,37 & 0,24 & 0,88 \\
\hline P305 & 647.700 & 1.719 .000 & 2.366 .700 & 0,27 & 1,00 \\
\hline \multicolumn{6}{|l|}{ Y305 } \\
\hline PDC1-PDC10 & - & - & - & - & 1,29 \\
\hline PDC1-PDC5 & - & - & - & - & 1,23 \\
\hline $\mathrm{PDCBI}^{1} Y T D B I$ & - & - & - & - & 1,27 \\
\hline
\end{tabular}

1 Produção dos controles bimensais $(1,4,7,10)$.

1 Test day milk yield bimonthly. 


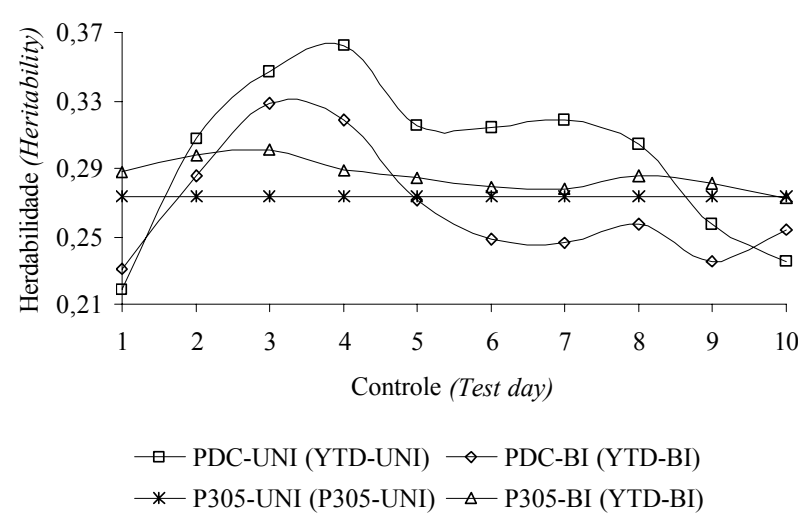

Figura 1 - Estimativas de herdabilidade obtidas, respectivamente, com modelos uni-caráter e bicaráter, para as produções de leite no dia do controle (PDC-UNI e PDC-BI) e para a produção de leite até 305 dias (P305-UNI e P305-BI) de primeiras lactações de vacas da raça Holandesa.

Figure 1 - Estimates of heritability obtained, respectively, by univariate and bivariate models, for test day yield (YTD-UNI and YTD-BI), and for 305-days milk yield (P305-UNI and P305-BI), of first lactation Holstein cows.
As correlações genéticas para as PDC variaram de 0,64 (entre PDC1 e PDC10) a 1 (entre PDC3 e $\mathrm{PDC} 7$ e entre PDC4 e PDC9), entretanto, as maiores freqüências foram para valores próximos ou iguais a 1 $(71 \% \geq 0,95$ e $47 \% \geq 0,98)$. As correlações entre as PDC a partir do sexto controle ( $\geq$ PDC6) foram todas iguais a 0,98. Entretanto, as correlações genéticas entre as PDC do início ( $\leq$ PDC3) e do final ( $\geq$ PDC 8 ) da lactação foram inferiores a 0,91 (Tabela 5). As correlações fenotípicas variaram de 0,27 (entre PDC1 e PDC10) a 0,78 (entre PDC3 e PDC4; entre PDC4 e PDC5; entre PDC5 e PDC6; entre PDC6 e PDC7; e entre PDC7 e PDC8) e apresentaram o mesmo comportamento das correlações genéticas, com valores superiores entre as PDC adjacentes (Tabela 5). As correlações residuais (resultados não apresentados) foram inferiores às demais (genéticas e fenotípicas), com valores variando de 0,69 (entre PDC5 e PDC7; entre PDC7 e PDC8; e entre PDC8 e PDC10) a 0,15 (entre PDC1 e PDC10). Estes resultados, com valores de

Tabela 4 - Estimativas dos componentes de variância genética aditiva $\left(\hat{\sigma}_{a}^{2}\right)$, residual $\left(\hat{\sigma}_{e}^{2}\right)$, fenotípica $\left(\hat{\sigma}_{p}^{2}\right)$ e de herdabilidades $\left(\hat{h}^{2}\right)$ para as produções de leite no dia do controle (PDC) e para a produção de leite até 305 dias (P305) de primeiras lactações de vacas da raça Holandesa, em análise bi-caráter

Table 4 - Estimates of genetic additive $\left(\hat{\sigma}_{a}^{2}\right)$, residual $\left(\hat{\sigma}_{e}^{2}\right)$, and phenotypic $\left(\hat{\sigma}_{p}^{2}\right)$, variance components and heritability $\left(\hat{h}^{2}\right)$ for test day milk yield (YTD) and for 305-days milk yield (Y305) of first lactation Holstein cows by bivariate model

\begin{tabular}{|c|c|c|c|c|c|c|c|c|}
\hline \multirow{2}{*}{$\begin{array}{l}\text { Controle } \\
\text { Test day }\end{array}$} & \multicolumn{8}{|c|}{$\begin{array}{l}\text { P305 } \\
Y 305\end{array}$} \\
\hline & $\hat{\sigma}_{a P D C}^{2}$ & $\hat{\sigma}_{e P D C}^{2}$ & $\hat{\sigma}_{p P D C}^{2}$ & $\hat{h}_{P D C}^{2}$ & $\hat{\sigma}_{a P 305}^{2}{ }^{1}$ & $\hat{\sigma}_{e P 305}^{2}$ & $\hat{\sigma}_{p P 305}^{2}$ & $\hat{h}_{P 305}^{2}$ \\
\hline PDC1 & 5,37 & 17,90 & 23,27 & 0,23 & 686,8 & 1695 & $2381,8^{1}$ & 0,29 \\
\hline YTD1 & 6,31 & 15,75 & 22,06 & 0,29 & 709,5 & 1673 & 2382,5 & 0,30 \\
\hline PDC2 & 7,32 & 14,94 & 22,26 & 0,33 & 717,3 & 1670 & 2387,3 & 0,30 \\
\hline PDC3 & 7,42 & 15,86 & 23,28 & 0,32 & 685,7 & 1684 & 2369,7 & 0,29 \\
\hline PDC4 & 6,56 & 17,66 & 24,22 & 0,27 & 670,8 & 1691 & 2361,8 & 0,28 \\
\hline PDC5 & 6,19 & 18,75 & 24,94 & 0,25 & 656,7 & 1700 & 2356,7 & 0,28 \\
\hline PDC6 & 6,31 & 19,31 & 25,62 & 0,25 & 656,4 & 1707 & 2363,4 & 0,28 \\
\hline PDC7 & 6,74 & 19,43 & 26,17 & 0,26 & 678,2 & 1699 & 2377,2 & 0,29 \\
\hline PDC8 & 6,21 & 20,16 & 26,37 & 0,24 & 668,6 & 1711 & 2379,6 & 0,28 \\
\hline PDC9 & 6,49 & 19,13 & 25,62 & 0,25 & 646,0 & 1727 & 2373,0 & 0,27 \\
\hline
\end{tabular}

${ }^{1}$ Estimativas expressas em $10^{3}$.

${ }^{1}$ Estimates expressed in $10^{3}$.

correlações genéticas superiores entre as produções dos controles consecutivos, assemelham-se aos obtidos por Meyer et al. (1989), Swalve (1995), Gadini (1997) e Olori et al. (1999).

As correlações genéticas entre as PDC e a P305 foram altas, variando de 0,86 (entre PDC1 e P305) a 0,99 (entre PDC3 e P305), com maiores valores entre as PDC do período intermediário da lactação e a P305
(Tabela 5). Dessa forma, se as decisões de descarte dos animais forem baseadas nas PDC da fase intermediária da lactação, que apresentam maiores valores de herdabilidade (Tabela 3) e maiores correlações genéticas com a P305 (Tabela 5), poderia se obter maiores ganhos genéticos na P305 que se decisões fossem tomadas com base nas PDC da fase inicial ou final da lactação. 
Tabela 5 - Estimativas de correlações genéticas (acima da diagonal), fenotípicas (abaixo da diagonal) entre as produções de leite no dia do controle (PDC) e a produção de leite até 305 dias (P305) de primeiras lactações de vacas da raça Holandesa

Table 5 - Estimates of genetic (above the diagonal), and phenotypic (below the diagonal) correlations between test day milk yield (YTD) and 305-days milk yield (Y305) of first lactation Holstein cows

\begin{tabular}{lccccccccccc}
\hline & PDC1 & PDC2 & PDC3 & PDC4 & PDC5 & PDC6 & PDC7 & PDC8 & PDC9 & PDC10 & P305 \\
\hline PDC1 & - & 0,96 & 0,96 & 0,95 & 0,93 & 0,92 & 0,90 & 0,84 & 0,75 & 0,64 & 0,86 \\
YTD1 & & & & & & & & & & & \\
PDC2 & 0,71 & - & 0,98 & 0,97 & 0,95 & 0,94 & 0,95 & 0,88 & 0,82 & 0,80 & 0,93 \\
PDC3 & 0,61 & 0,77 & - & 0,98 & 0,97 & 0,96 & 1,00 & 0,91 & 0,86 & 0,85 & 0,99 \\
PDC4 & 0,53 & 0,69 & 0,78 & - & 0,98 & 0,98 & 0,97 & 0,97 & 1,00 & 0,96 & 0,97 \\
PDC5 & 0,48 & 0,63 & 0,71 & 0,78 & - & 0,98 & 0,98 & 0,98 & 0,98 & 0,98 & 0,97 \\
PDC6 & 0,44 & 0,58 & 0,66 & 0,71 & 0,78 & - & 0,98 & 0,98 & 0,98 & 0,98 & 0,97 \\
PDC7 & 0,40 & 0,53 & 0,61 & 0,65 & 0,71 & 0,78 & - & 0,98 & 0,98 & 0,98 & 0,93 \\
PDC8 & 0,37 & 0,49 & 0,56 & 0,60 & 0,65 & 0,71 & 0,78 & - & 0,98 & 0,98 & 0,96 \\
PDC9 & 0,33 & 0,44 & 0,51 & 0,54 & 0,58 & 0,62 & 0,68 & 0,77 & - & 0,98 & 0,95 \\
PDC10 & 0,27 & 0,38 & 0,46 & 0,48 & 0,51 & 0,55 & 0,60 & 0,68 & 0,76 & - & 0,96 \\
P305 & 0,57 & 0,68 & 0,74 & 0,77 & 0,81 & 0,83 & 0,83 & 0,83 & 0,80 & 0,76 & - \\
Y305 & & & & & & & & & & & \\
\hline
\end{tabular}

Tabela 6 - Estimativas de covariâncias genética (acima da diagonal) e fenotípica (abaixo da diagonal) entre as produções de leite no dia do controle (PDC) de primeiras lactações de vacas da raça Holandesa

Table 6 - Estimates of genetic (above the diagonal) e phenotypic (below the diagonal) covariance between test day milk yield (YTD) of first lactation Holstein cows

\begin{tabular}{lcccccccccc}
\hline & PDC1 & PDC2 & PDC3 & PDC4 & PDC5 & PDC6 & PDC7 & PDC8 & PDC9 & PDC10 \\
\hline PDC1 & - & 5,94 & 6,37 & 6,68 & 5,81 & 7,51 & 5,10 & 5,21 & 3,98 & 3,29 \\
YTD1 & & & & & & & & & & \\
PDC2 & 9,93 & - & 7,71 & 7,34 & 6,82 & 6,50 & 6,09 & 5,95 & 4,88 & 4,78 \\
PDC3 & 7,21 & 9,77 & - & 7,96 & 7,66 & 7,21 & 7,30 & 6,64 & 5,61 & 5,57 \\
PDC4 & 5,31 & 7,67 & 9,20 & - & 7,94 & 7,67 & 7,47 & 7,51 & 7,13 & 6,69 \\
PDC5 & 4,99 & 6,87 & 8,08 & 9,37 & - & 6,82 & 6,69 & 7,25 & 6,43 & 6,50 \\
PDC6 & 4,03 & 5,97 & 7,21 & 7,90 & 10,15 & - & 6,96 & 7,33 & 6,43 & 6,77 \\
PDC7 & 3,74 & 5,35 & 6,03 & 6,76 & 8,63 & 9,78 & - & 6,53 & 6,23 & 6,42 \\
PDC8 & 2,82 & 4,43 & 5,56 & 5,50 & 6,75 & 7,94 & 9,98 & - & 6,14 & 6,09 \\
PDC9 & 3,13 & 4,40 & 5,46 & 4,57 & 5,60 & 6,87 & 8,23 & 10,14 & - & 5,28 \\
PDC10 & 2,52 & 3,20 & 4,22 & 3,64 & 4,49 & 4,94 & 6,23 & 8,28 & 10,86 & - \\
\hline
\end{tabular}

Assim, maior ganho genético na P305 pode ser obtido por seleção indireta baseada nas PDC3 a PDC8, particularmente nas PDC3 e PDC4, que apresentam maior eficiência relativa que a seleção direta para a P305 (Tabela 3). A seleção realizada com base nas PDC poderia resultar em ganhos $29 \%$ superiores aos obtidos da seleção sob P305 (utilizando os 10 controles), além de possibilitar avaliações com produções parciais (cinco primeiros controles) e controles esparsos (controles bimensais) ainda com ganhos superiores aos esperados para $\mathrm{P} 305$ ( 23 e 27\%, respectivamente, para os cinco primeiros controles e para controles bimensais) (Tabela 3). Ribas \& Perez (1990) relataram maior ER para o PDC5 e PDC6 em estudo como PDC de primeiras lactações de vacas Holandesas.
Estimativas de covariâncias genéticas e fenotípicas entre as PDC são apresentadas na Tabela 6.

As covariâncias genéticas e fenotípicas entre as PDC foram maiores particularmente na fase intermediária da lactação, diminuindo em magnitude à medida em que os mesmos se distanciavam. Considerando-se que as estimativas de correlações genéticas entre as PDC foram altas e próximas à unidade entre a maioria das PDC $(71 \%>0,95)$, pode-se assumir que as correlações não diferem de 1 entre as PDC. Este fato sugere que um modelo de repetibilidade (Rekaya et al., 1999; Melo et al., 2002) poderia ser utilizado para o ajuste das PDC, visto que o mesmo é mais parcimonioso, pois necessita estimar apenas três componentes de covariância, e, portanto, é menos exigente 
em termos de demanda computacional e tempo de processamento.

Apesar de maior simplicidade e eventual facilidade de análise ao assumir variâncias constantes e correlações iguais a um entre as PDC, o modelo de repetibilidade não considera a forma da curva da lactação. Contudo, ajustar todas as produções disponíveis ao longo da lactação em um modelo multicaráter é mais difícil em razão do maior número de parâmetros a serem estimados. Neste contexto, os modelos de regressão aleatória, com submodelos que permitem o ajuste da forma da curva da lactação e consideram a estrutura de covariância entre as PDC, caracterizam-se como uma interessante e promissora alternativa para o ajuste das PDC (Brotherstone et al., 2000; Pool et al., 2000; Kettunen et al., 2000; Pool \& Meuwissen, 1999; Jakobsen et al., 2002) devendo ser investigados em estudos futuros.

Os seguintes autovalores $\left(\lambda_{i}\right) 60,06 ; 3,01 ; 0,52$; 0,$28 ; 0,21 ; 0,13 ; 0,10 ;-0,02,-0,22 ;-0,94$ e 76,$44 ; 24,50$; 13,$92 ; 8,31 ; 5,75 ; 4,81 ; 4,56 ; 4,14 ; 3,74 ; 3,67$ são produzidos, respectivamente, pela decomposição canônica da matriz de covariância genética e fenotípica. Pela decomposição canônica da matriz G, tem-se que os dois primeiros autovalores contabilizam $96 \%$ da variância genética (desconsiderando o sinal dos demais). Todavia, para a matriz $\mathrm{R}$, são necessários os quatro primeiros autovalores para contabilizar o mesmo percentual da variância residual. Os autovetores associados a estes autovalores (dois primeiros de $\mathrm{G}$ e quatro primeiros de R) são apresentados na Figuras 2 e 3 , respectivamente.

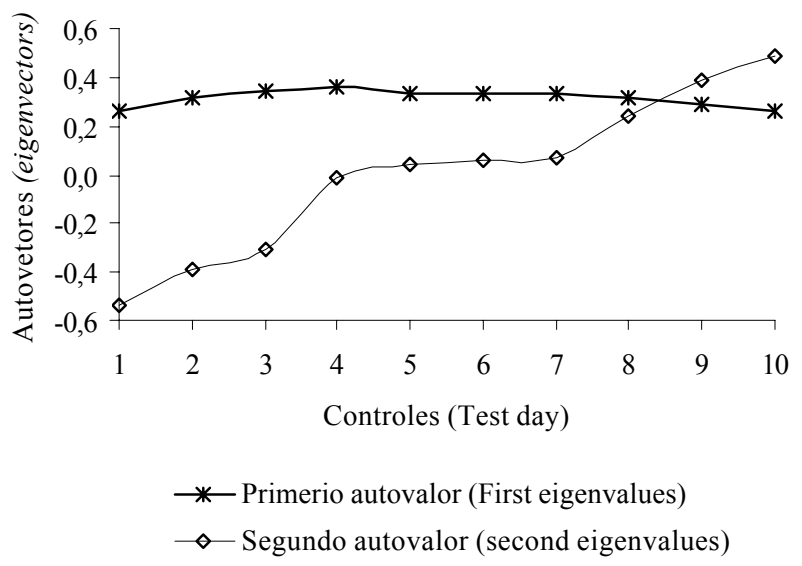

Figura 2 - Autovetores associados aos dois primeiros autovalores da matriz de covariância genética.

Figure 2 - Eigenvectors associated with the two first eigenvalues of the matrix covariance genetic.

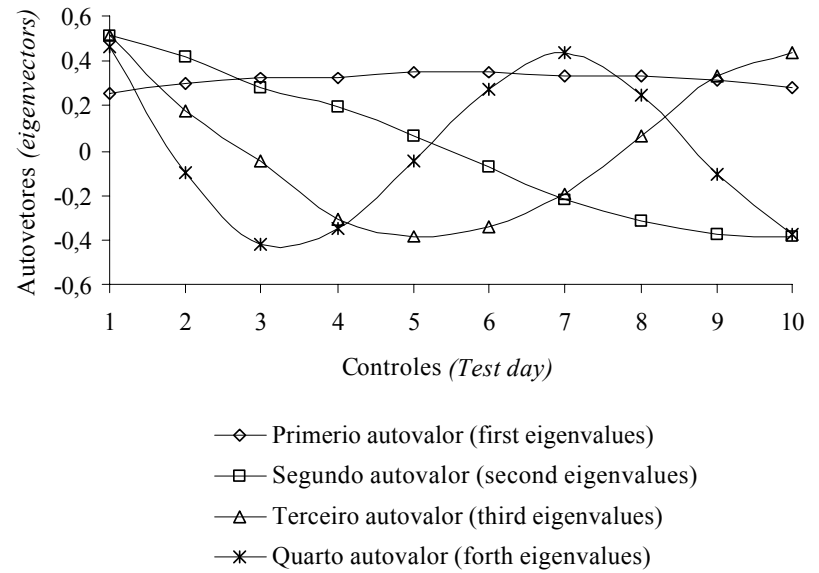

Figura 3 - Autovetores associados aos quatro primeiros autovalores da matriz de covariância residual.

Figure 3 - Eigenvectors associated with the four first eigenvalues of the matrix covariance residual.

Nota-se que os dois primeiros autovetores da matriz de covariância genética (Figura 2) correspondem, aproximadamente, aos efeitos constante e linear de dias em lactação e os quatros primeiros autovetores da matriz de covariância residual (Figura 3) aos efeitos constante, linear, quadrático e cúbico. Pode-se, portanto, sugerir que um modelo de regressão aleatória utilizando um polinômio de primeira ordem (efeitos constante e linear) poderia ser suficiente para ajustar o efeito genético no modelo. Todavia, ordens superiores (terceira ordem) seriam necessárias para ajustar os efeitos de ambiente.

\section{Conclusões}

A não-inclusão das covariáveis que descrevem a curva de lactação no modelo de ajuste das PDC, sob modelo de repetibilidade, resultou em estimativas inflacionadas da variância genética aditiva.

As PDC da fase intermediária da lactação podem ser utilizadas como critérios de seleção dos animais como forma de obter predição mais confiável do valor genético dos animais, particularmente aquelas que têm maiores herdabilidade (produções dos controles dois a oito) que a da produção de leite até 305 dias e são altamente correlacionadas com esta. Nesta situação, a seleção indireta baseada nas PDC pode ser mais eficiente que a seleção direta para P305. Assim, a utilização das PDC poderia possibilitar avaliações por produções parciais (e.g. PDC1 a PDC5) ou controles esparsos (e.g. bimensais) e ainda resultar em ganhos superires a $20 \%$ em relação a P305. 
Estimativa de correlações genéticas altas e próximas à unidade entre a maioria das PDC sugerem que, eventualmente, pode-se assumir correlação igual a um entre as PDC e ajustar um modelo de repetibilidade, que é mais parcimonioso e menos exigente em termos de demanda computacional.

A decomposição canônica das matrizes de covariâncias genética e residual mostrou que um modelo de regressão aleatória utilizando um polinômio de primeira ordem pode ser suficiente para ajustar o efeito genético. Contudo, um polinômio de ordem três é necessário para ajustar os efeitos de ambiente. Neste contexto, é importante verificar o ajuste destes modelos em trabalhos futuros para se concluir sobre a melhor utilização das PDC na avaliação genética da raça Holandesa no Brasil.

\section{Agradecimento}

Ao professor Ignacy Misztal, da Universiy of Georgia, EUA, por disponibilizar os programas computacionais RENUMMAT3 e REMLF90; à ABCBRH, ao Prodtab; e aos revisores, pelas valiosas sugestões apresentadas.

\section{Literatura Citada}

BROTHERSTONE, S.; WHITE, I.; MEYER, K. Genetic modeling of dairy milk yield using orthogonal polynomial and parametric curves. Animal Science, n.70, p.407-415, 2000.

COSTA, C.N. An investigation into heterogeneity of variance for milk and fat yields of Holstein cows in Brazilian herd environments. Genetic and Molecular Biology, v.22, n.3, p.375-381, 1999.

COSTA, C.N.; BLAKE, R.W.; POLLAK, E.J. et al. Genetic analysis of Holstein cattle populations in Brazil and United States. Journal of Dairy Science, v.83, n.12, p.2963-2974, 2000.

DANELL, B. Studies on lactation yield and individual test-day yields of Swedish dairy cow. II. Estimates of genetic and phenotypic parameters. Acta Agriculturae Scandinavica, v.32, p.103-114, 1982.

EL FARO, L. Estimação de componentes de (co)variância para produção de leite no dia do controle de primeiras lactações de vacas Caracu, aplicando-se "test day models" de dimensão finita e modelos de regressão aleatória. Jaboticabal: Universidade Estadual Paulista, 2002. 102p. Tese (Doutorado) - Universidade Estadual Paulista.

FERREIRA, W.J. Avaliação genética de bovinos da raça Holandesa usando a produção de leite no dia do controle. Revista da Sociedade Brasileira de Zootecnia, v.32, n.2, p.295-303, 2003.

FREITAS, A. F.; DURÃES, M.C.; VALENTE, J. et al. Parâmetros genéticos para produção de leite e gordura nas três primeiras lactações de vacas Holandesas. Revista Brasileira de Zootecnia, v.30, n.3, p.709-713, 2001.
GADINI, C.H. Genetic evaluation of test day production traits and somatic cell scores. Licoln: University of Nebrask, 1997. Thesis (Ph.D) - University of Nebrask, 1997.

GADINI, H.C.; KEOWN, J.F.; Van VLECK, L.D. Genetic parameters of test day milk, fat and protein yields. Genetic parameters of test-day milk yield of Holstein cows (compact disc). In: WORLD CONGRESS OF GENETICS APPLIED LIVESTOCK PRODUCTION, 6., 2002, Armidale. Proceedings... Armidale: University of New England, 1998.

JAKOBSEN, J.H.; MADSEN, P.; JENSEN, J. et al. Genetic parameters for milk production and persistency for Danish Holsteins estimated in random regression models using REML. Journal of Dairy Science, v.85, n.6, p.1607-1616, 2002.

KETTUNEN, A.; MÄNTYSAARI, E.A.; PÖSÖ, J. Estimation of genetic parameters for daily milk yield of primiparous Ayrshire cows by random regression test-day models. Livestock Production Science, v.66, p.251-261. 2000.

KETTUNEN, A.; MÄNTYSAARI, E.A.; STRANDÉN, I. et al. Estimation of genetic parameters for first lactation test day milk production using random regression models (compact disc). In: WORLD CONGRESS OF GENETICS APPLIED LIVESTOCK PRODUCTION, 6., 1998, Armidale. Proceedings... Armidale: University of New England, 1998.

MACHADO, S.G. Parâmetros genéticos e de ambiente da produção de leite no dia do controle da primeira lactação de vacas da raça Holandesa. Jaboticabal: Universidade Estadual Paulista, 1997. 76p. Dissertação (Mestrado) Universidade Estadual Paulista, 1997.

MACHADO, S.G.; FREITAS, M.A.R.; GADINI, C.H. Genetic parameters of test-day milk yield of Holstein cows (compact disc). In: WORLD CONGRESS OF GENETICS APPLIED LIVESTOCK PRODUCTION, 6., Armidale, 1998. Proceedings... Armidale: University of New England, 1998.

MACHADO, S.G.; FREITAS, M.A.R.; GADINI, C.H. Genetic parameters of test day milk yields of Holstein cows. Genetic and Molecular Biology, v.22, n.3, p.383-386. 1999.

MELO, C.M.R.; COSTA, C.N.; PACKER, I.U. et al. Parâmetros genéticos para as produções de leite de controles individuais e total de primeiras lactações de vacas da raça Holandesa. In: REUNIÃO ANUAL DA SOCIEDADE BRASILEIRA DE ZOOTECNIA, 39., 2002, Recife. Anais... Recife: Sociedade Brasileira de Zootecnia, 2002. CD-ROM

MEYER, K.; GRASER, H.U.; HAMMOND, K. Estimates of genetic parameters for first lactation test day production of Australian Black and White cows. Livestock Production Science, v.21, p.177-199, 1989.

MISZTAL, I. 1999. REMLF90: manual. ftp://nce.ads.uga.edu/ pub/ignacy/blupf90/ docs/remlf90.pdf. (05 de jan. 2001).

OLORI, V.E. Utilization of daily milk records in genetic evaluation of dairy cattle. Edinburgh, 1997. 1v. Thesis (Ph.D) - University of Edinburgh, 1997.

OLORI, V.E.; HILL, W.G.; McGUIRK, B.J. et al. Estimating variance e components for test day milk records by restricted maximum likelihood with a random regression animal model. Livestock Production Science, v.61, n., p.53-63, 1999.

POOL, M.H.; JANSS, L.L.G.; MEUWISSEN, T.H.E. Genetic parameters of Legendre polynomials for first parity lactation curves. Journal of Dairy Science, v.83, n.11, p.2640-2649, 2000.

POOL, M.H.; MEUWISSEN, T.H.E. Prediction of daily milk yields from a limited number of test days using test day models. Journal of Dairy Science, v.82, n.7, p.1555$1564,1999$. 
PÖSÖ, J.; MÄNTYSAARI, E.A.; LIDAUER, M. et al. mpirical bias in the pedigree indices of heifers evaluated using test day models (compact disc). In: WORLD CONGRESS OF GENETICS APPLIED LIVESTOCK PRODUCTION, 6., 1998, Armidale. Proceedings... Armidale: University of New England, 1998.

PTAK, E.; SCHAEFFER, L.R. Use of test day yields for genetic evaluation of dairy sires and cows. Livestock Production Science, v.34, n.1-2, p.23-34, 1993.

REENTS, R.; DEKKERS, J.C.M.; SCHAEFFER, L.R. Genetic evaluation for somatic cell score with a test day model for multiple lactations. Journal of Dairy Science, v.78, n.12, p.2858-2870, 1995.

REENTS, R.; JAMROZICK, J.; SCHAEFFER, L.R. et al. Estimation of genetic parameters for test day records of somatic cell score. Journal of Dairy Science, v.78, n.12, p.2847-2857, 1995.

REKAYA, R.; BEJAR, R.; CARABAÑO, M.J. et al. Genetic parameters for test day measurements in Spanish HolsteinFriesian. In: INTERBULL, 11., 1995, Prague. Proceedings... Prague: Czech Republic, 1995. p.1-8 (Bulletin 11).

REKAYA, R.; CARABAÑO, M.J.; TORO, M.A. Use de test day yields for the genetic evaluation of production traits in Holstein-Friesian cattle. Livestock Production Science, v.57, p.203-217, 1999.

RIBAS, M.; PEREZ, B. Monthly test day milk records and yield at 244 days. II. Genetic parameters in first lactation. Cuban Journal of Agricultural Science, v.24, n.2, p.129-144, 1990.
STRABEL, T.; SZWACZKOUSKI, T. Additive genetic and permanent environmental variance components for test day milk yields in Black-white cattle. Livestock Production Science, v.48, p.91-98, 1997.

SWALVE, H.H. The effect of test day models on the estimation of genetic parameters and breeding values for dairy yield traits. Journal of Dairy Science, v.78, n.4, p.929-938, 1995.

TORRES, R.A.; BERGMAN, J.A.G.; COSTA, C.N. et al. Ajustamento para heterogeneidade de variância para a produção de leite entre rebanhos da raça Holandesa no Brasil. Revista Brasileira de Zootecnia, v.28, n.2, p.295-305. 1999.

VAN TASSELL, C.P.; QUAAS, R.L.; EVERETT, R.W. Parameter estimates for 305-day residual records. Journal of Dairy Science, v.75, suppl. 1, p.251, 1992.

VARGAS, B.; PEREZ, E.; Van ARENDONK, J.A.M. Analysis of test day yield data of Costa Rican dairy cattle. Journal of Dairy Science, v.81, n.1, p.225-271, 1998.

VISSCHER, P.M.; GODDARD, M.E. Genetic parameters for milk yield, survival, workability and type traits for Australian dairy cattle. Journal of Dairy Science, v.78, n.1, p.205-220, 1995.

WIGGANS, G.R.; GODDARD, M.E. A computationally feasible test day model for genetic evaluation of traits in the United States. Journal of Dairy Science, v.80, n.8, p.1795-1800, 1997.

Recebido em: 06/02/03

Aceito em: 29/10/04 